\title{
Preparation and Investigation of the Microtribological Properties of Graphene Oxide and Graphene Films via Electrostatic Layer-by-Layer Self-Assembly
}

\author{
Yongshou Hu, ${ }^{1}$ Haibing Ma, ${ }^{1}$ Wei Liu, ${ }^{2}$ Qianqian Lin, ${ }^{2}$ and Bin Liu ${ }^{2}$ \\ ${ }^{1}$ Department of Stomatology, Traditional Chinese Medicine Hospital of Gansu Province, Lanzhou 730050, China \\ ${ }^{2}$ School of Nuclear Science and Technology, Stomatology, Lanzhou University, Lanzhou 730000, China \\ Correspondence should be addressed to Bin Liu; liubkq@lzu.edu.cn
}

Received 17 September 2014; Accepted 22 December 2014

Academic Editor: Wenwen Liu

Copyright ( $\odot 2015$ Yongshou Hu et al. This is an open access article distributed under the Creative Commons Attribution License, which permits unrestricted use, distribution, and reproduction in any medium, provided the original work is properly cited.

Graphene oxide (GO) films with controlled layers, deposited on single-crystal silicon substrates, were prepared by electrostatic self-assembly of negatively charged GO sheets. Afterward, graphene films were prepared by liquid-phase reduction of as-prepared GO films using hydrazine hydrate. The microstructures and microtribological properties of the samples were studied using Xray photoelectron spectroscopy, Raman spectroscopy, X-ray diffraction, UV-vis absorption spectroscopy, water contact angle measurement, and atomic force microscopy. It is found that, whether GO films or graphene films, the adhesion force and the coefficients of friction both show strong dependence on the number of self-assembled layers, which both allow a downward trend as the number of self-assembled layers increases due to the interlayer sliding and the puckering effect when the tip slipped across the top surface of the films. Moreover, in comparison with the GO films with the same self-assembled layers, the graphene films possess lower adhesion force and coefficient of friction attributed to the difference of surface functional groups.

\section{Introduction}

With the rapid development of science and technology, especially of nanotechnology, micromechanical and highdensity magnetic recording systems, the study of microtribology has attracted wide attention. In theoretical research, microtribology requires exploration deep into the atomic and molecular scale to extensively reveal the phenomena of friction processes and plays an important role in perfecting the theory of tribology [1]. In applied research, the continual emergence of advanced technologies, such as magnetic recording systems and microelectromechanical systems (MEMs), not only brings new challenges for traditional tribology and lubrication technology, but also prompts studies on friction at the microscale and development of nanolubrication technology [2].

Although MEMs are known for their superior performance and low unit cost [3], their large surface areato-volume ratios result in serious adhesive and frictional problems that limit their operations [4]. Also, conventional lubricants are no longer particularly desirable due to the microsize of MEMs components. Reports on improvements in the microtribological performance of MEMs are divided into two aspects: (i) preparation of a super-hard surface coating on a silicon substrate via ion implantation or thin film deposition to enhance wear resistance, and (ii) deposition of a soft coating such as Langmuir-Blodgett (LB) film to reduce the coefficient of friction $(\mu)$ of the silicon surface and further improve friction and wear properties. However, many super-hard coatings, such as $\mathrm{SiC}$, diamond, and diamond-like carbon, are not easy to prepare, and LB films are limited by their poor stability, poor ability to resist external interference, and short life. For the above reasons, exploration of new methods to prepare protection films with good frictionreduction and wear-resistance is of utmost importance. As we know, an approach to preparing protection films with obvious wear-resistance behavior is the self-assembly technique [57]. Compared with other techniques, self-assembly has some special advantages, such as simple preparation, controlled film thickness, thermodynamic stability, and independence 
from the size and shape of the base, giving it broad application prospects in the field of tribology [8]. Lio et al. [9] prepared alkanethiols and alkyl silanes self-assembly monolayers (SAMs) with different alkyl chain lengths and found that short-chain SAMs exhibit higher $\mu$ than longchain SAMs. Beake and Leggett [10] studied mixed SAM systems composed of short- and long-chain alkanethiols and they found that the $\mu$ varies substantially with monolayer composition, especially of changes in the alkyl chain. Huang et al. [11] prepared self-assembled multilayer films consisted of nitrocontaining diazoresin and polysodium $\mathrm{p}$ styrenesulfonate (NDR-PSS), and the study of their tribological behaviors showed that the 10-bilayer film exhibits better load-carrying and antiwear abilities than 1-bilayer film. Gao and $\mathrm{Yu}$ [12] believed that the tribological behaviors of multilayer polyelectrolyte films are closely related to the layer numbers. Ren et al. [13] prepared a stearic acid and 3aminopropyl triethoxysilane (STA-APS) dual-layer film on a single-crystal silicon substrate via chemical self-assembly, and the tribological experiment showed that the STA-APS film has good adhesive-resistance and friction-reduction in both microscopic and macroscopic levels.

Graphene, a 2-dimensional atomic thin layer of carbon nanostructure, exhibits notable electronic, thermal, chemical, and mechanical properties, as well as unrivaled mechanical stiffness and strength [14]. Thus, it is considered highly applicable in next-generation electronic devices and MEMs [15, 16]. Lee et al. [17] found that the frictional force on graphene is smaller than that on the silicon oxide substrate, while larger than that on bulk graphite. They also compared the nanoscale friction characteristics of atomically thin sheets (including graphene, molybdenum disulfide, niobium diselenide, and hexagonal boron nitride) exfoliated onto weakly adherent silicon oxide substrates with those of their bulk counterparts and revealed that friction force of each sheet-like material monotonically increases with the number of layers decreasing [18].

However, it should be mentioned that realization of graphene application in MEMs is severely restricted by the difficulty of obtaining a stable graphene film on a device surface via exfoliation-sticking and common solution processes. The key defect is attributed to the lack of active groups on the surface of graphene, resulting in a very week adhesion to substrate. Fortunately, the existence of abundant oxygenous groups on graphene oxide (GO) sheets makes the self-assembly technique feasible, to prepare graphene films with good adhesion to substrates [19-21]. Ou et al. [22] revealed that assembled reduced graphene oxide (RGO) monolayer film on silicon substrate possesses good friction reduction and antiwear abilities due to its intrinsic structure.

In this paper, a facile preparation of graphene multilayer films with controlled layers, deposited on silicon substrates, was realized by electrostatic self-assembly of negatively charged GO sheets followed by liquid-phase reduction using hydrazine hydrate. An investigation of the relationship between the microtribological characteristics and the number of self-assembled layers was employed in-depth. Also, comparisons of the microtribological properties between self-assembled GO and RGO multilayer films were carried out as well.

\section{Experimental}

2.1. Materials. Polished single-crystal silicon (111) wafers, obtained from GRINM Semiconductor Materials Co., Ltd., Beijing, with a surface roughness of about $0.4 \mathrm{~nm}$ after cleaning, were used as the substrates. Poly(diallyldimethylammonium chloride) (PDDA, $\mathrm{Mw}=400,000-500,000$, $20 \mathrm{wt} . \%$ solution in water) was obtained from Sigma-Aldrich. GO was synthesized using a modified Hummers method $[23,24]$. Other reagents were of analytical purity and used as received. Ultrapure water $\left(18 \mathrm{M} \Omega \cdot \mathrm{cm}^{-1}\right)$ was used throughout the experiment.

2.2. Preparation of GO and Graphene Multilayer Films. Silicon substrates were cleaned and hydroxylated by immersing in a piranha solution (a mixture of $7: 3(\mathrm{v} / \mathrm{v}) 98 \% \mathrm{H}_{2} \mathrm{SO}_{4}$ and $30 \% \mathrm{H}_{2} \mathrm{O}_{2}$ ) at $30^{\circ} \mathrm{C}$ for $20 \mathrm{~min}$. The substrates were then fully rinsed with ultrapure water, placed into a $0.2 \mathrm{wt} \%$ PDDA aqueous solution, and then held for $15 \mathrm{~min}$. A monolayer of PDDA was thus formed on each silicon surface. At the same time, a homogeneous GO aqueous suspension $(0.5 \mathrm{mg} / \mathrm{mL})$ was prepared by sonication and centrifugation. After rinsing with ultrapure water, a PDDA-coated silicon substrate was immersed in the GO suspension and kept for 15 min. A monolayer of GO was presumably produced on top of the PDDA film via electrostatic interaction between the negatively charged GO and the positively charged PDDA. By repeating the above steps, GO multilayer films with the desired layers were obtained. Subsequently, RGO multilayer films were prepared by the reduction of as-prepared GO films with hydrazine hydrate as described elsewhere [25]. In order to investigate the microstructural change of GO through the hydrazine hydrate reduction, powdery RGO was prepared as well.

Moreover, in order to certify that the graphene films with gradually increased thickness can be prepared by repeating the electrostatic layer-by-layer (LBL) self-assembly; RGO multilayer films on indium tin oxide (ITO) glass substrates were prepared according to the same LBL self-assembly followed by the hydrazine hydrate reduction. Before the selfassembly experiments, each ITO substrate was cleaned with a $3.95 \mathrm{wt}$ \% ethanol solution of potassium hydroxide for $5 \mathrm{~min}$ using ultrasonication, thoroughly rinsed with ultrapure water and ethanol and was ready to use.

2.3. Characterization. The surface morphologies of the RGO sheets and the assembled GO and RGO films were investigated by a Nanoscope III a multimode atomic force microscope (AFM, Veeco) in tapping mode. The XPS measurements of powdery GO and RGO were performed on a PerkinElmer PHI-5702 multifunctional X-ray photoelectron spectroscope (Physical Electronics, USA), using Al-K $\alpha$ radiation (photon energy $1476.6 \mathrm{eV}$ ) as the excitation source and the binding energy of $\mathrm{Au}\left(\mathrm{Au} 4 \mathrm{f}_{7 / 2}: 84.00 \mathrm{eV}\right)$ as the reference. The crystallographic structures of the powdery samples were determined by a powder X-ray diffraction system (XRD, 
TTR-III) equipped with CuKa radiation $(k=0.15406 \mathrm{~nm})$. Raman spectroscopy (Lab RAMHR800, Horiba, Hobin Yvon, France, $532 \mathrm{~nm}$ laser excitation) was employed to characterize the microstructure of the film samples. UV-vis absorption spectra of the RGO multilayer films were monitored with a Hitachi U-2010 spectrophotometer. The water contact angle measurements of the film samples were carried out using the "sitting drop" method in atmospheric conditions at room temperature. Each measurement was repeated 10 times and reproducibility was never worse than $\pm 2^{\circ}$. The results of measurements were treated statistically.

2.4. Microtribological Properties of GO and Graphene Multilayer Films. The microtribological behavior was characterized with the same AFM/FFM instrument controlled by RHK electronics (RHK Technology, Rochester Hills, MI, USA) under contact mode. Mica was used to calibrate the photodetector sensitivity by measuring a force curve. Relative to the very flexible lever, the stiffness of the mica was sufficiently large; it was assumed that all deflection during the force measurement would occur in the lever. Under these circumstances, the photodetector sensitivity was the gradient of a plot of photodetector signals versus displacement while measuring repulsive forces. Commercially available $\mathrm{Si}_{3} \mathrm{~N}_{4}$ cantilevers/tips with a nominal force constant of $2.0 \mathrm{nN} \cdot \mathrm{m}^{-1}$ were employed. Friction force measurements were recorded in scope mode by obtaining friction loops from six separate areas on the monolayer surfaces. Construction of friction load plots was accomplished by varying the normal load applied to the tip. Data of $\mu$ were standardized using an internal normalization procedure previously described elsewhere $[26,27]$. A single tip was used to acquire a complete set of data for an entire series of samples, and the gradients of the friction-load plots were used to obtain $\mu$.

To obtain the adhesive force between the AFM tip and the film surface, the force distance curve was recorded and the pull-off force was assumed as the adhesive force, which was given by $F=K_{c} \Delta Z_{p}$, where $K_{c}$ is the force constant of the cantilever, and $\Delta Z_{p}$ is the vertical displacement of the piezotube, that is, the deflection of the cantilever $[28,29]$. The displacement of the retraction part from the approach part, caused by the hysteresis of the piezotube, was adjusted. To reduce the statistical error, we measured the adhesion force at many different locations on a sample surface. All the friction and adhesion tests were conducted at room temperature of $21^{\circ} \mathrm{C}$ and a relative humidity of $25 \%$.

\section{Results and Discussion}

As Figure 1 shows, the AFM image and its corresponding height profile show that an individual RGO sheet on a silicon wafer surface has a thickness of $0.9 \mathrm{~nm}$, which is accordant with the value for single-layer $\operatorname{RGO}$ sheet $[25,30]$. The measured thickness of the RGO sheet is much larger than the theoretical value of graphene layer $(0.34 \mathrm{~nm})$, which is due to the fact that a small number of oxygenous groups still remain on the surface of the RGO sheet after chemical reduction.

The Cls XPS spectra of GO and RGO sheets are shown in Figure 2. The Cls XPS spectrum of GO can be fitted into

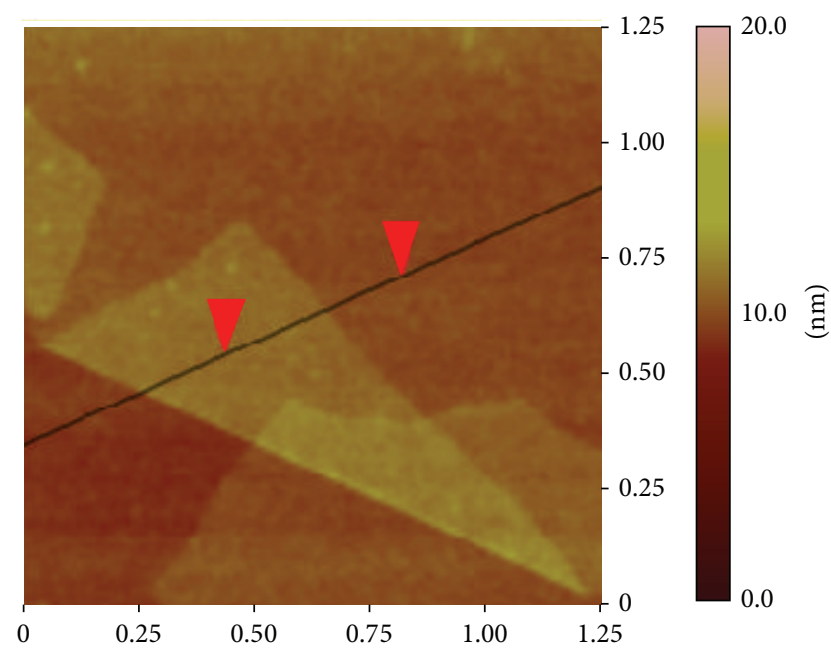

(a)

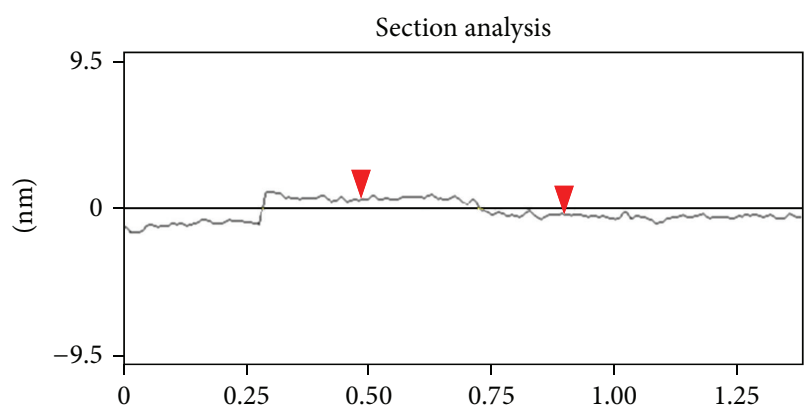

(b)

FIgURE 1: AFM image and the height profile of the RGO sheets.

four components [31]: non-oxygenated carbon at $284.8 \mathrm{eV}$, carbon in $\mathrm{C}-\mathrm{O}$ at $286.2 \mathrm{eV}$, carbonyl carbon $(\mathrm{C}=\mathrm{O}, 287.9 \mathrm{eV})$, and carboxylate carbon $(\mathrm{O}-\mathrm{C}=\mathrm{O}, 289.0 \mathrm{eV})$. In contrast to the Cls XPS spectrum of GO, the spectrum of RGO shows the presence of the same functionalities but with much smaller intensities, indicating that most of the oxygen functional groups were removed by reduction with hydrazine hydrate.

Structural difference between GO and RGO was further evidenced from the XRD measurements. As shown in Figure 3, the XRD pattern of GO has a diffraction peak at $12.1^{\circ}$, corresponding to water-bound intercalated oxidized graphite [32]. For the XRD pattern of RGO, the diffraction peaks at $24.5^{\circ}$ and $42.8^{\circ}$ are attributed to the graphite-like structures (002) and (100), respectively. This result indicates a relatively complete reduction from GO to RGO.

Figure 4 shows the Raman spectra of self-assembled GO and RGO multilayer films with the same layers of 20 . Two spectra both exhibit two intense peaks at $1328 \mathrm{~cm}^{-1}$ and $1595 \mathrm{~cm}^{-1}$, corresponding to the $\mathrm{D}$ and $\mathrm{G}$ bands, respectively. $2 \mathrm{D}$ and $\mathrm{D}+\mathrm{G}$ bands are also observed [33]. The $\mathrm{G}$ band is related to the vibration of $\mathrm{sp}^{2}$-hybridized carbon, whereas the $\mathrm{D}$ band corresponds to the $\mathrm{sp}^{2}$-hybridized carbon with defects associated with vacancies, disorder, and grain boundaries [34]. The intensity ratio of $I_{\mathrm{D}} / I_{\mathrm{G}}$ in RGO spectrum (1.11) obviously increases compared with that in GO spectrum 


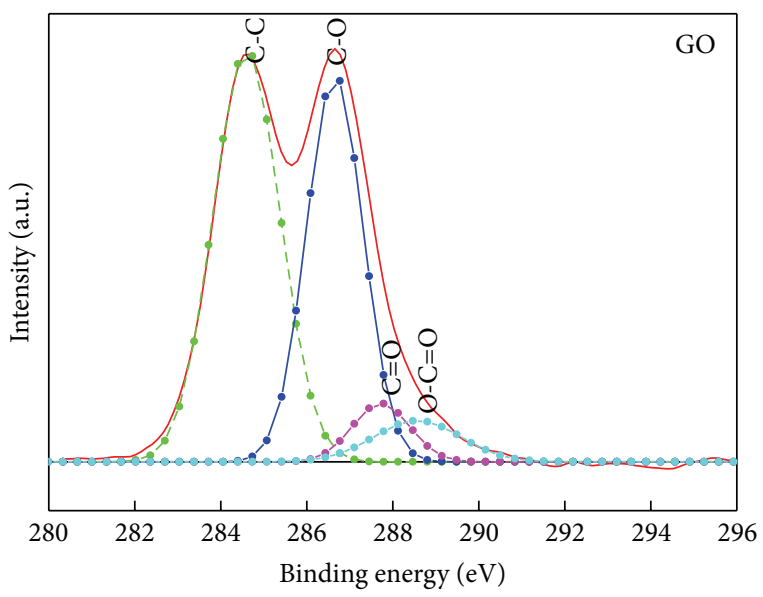

(a)

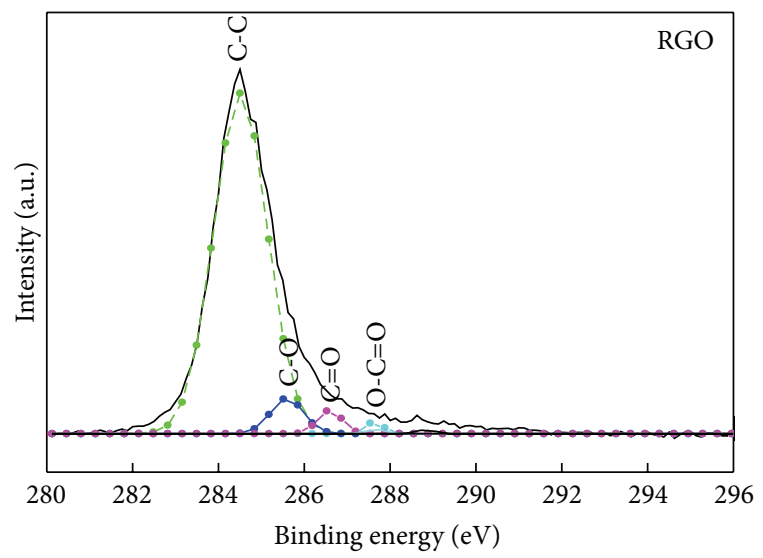

(b)

FIgUre 2: The C 1s XPS spectra of the GO (a) and RGO (b) sheets.

(1.01), indicating the realization of deoxygenation in chemical RGO film [25]. Moreover, in contrast to the ratio between the $2 \mathrm{D}$ and $\mathrm{G}$ bands, $I_{2 \mathrm{D}} / I_{\mathrm{G}}$, of the GO films, the ratio of $I_{2 \mathrm{D}} / I_{\mathrm{G}}$ for RGO increases due to the recovering of $\mathrm{sp}^{2} \mathrm{C}=\mathrm{C}$ bonds.

Figure 5 shows the UV-vis absorption spectra of RGO multilayer films on ITO glass substrates. The inset shows the nonlinear relationship between the absorbance of RGO films at $500 \mathrm{~nm}$ and the number of self-assembled layers. The absorbance at $500 \mathrm{~nm}$ nonlinearly increases with the increase of the self-assembled layers, suggesting that the RGO multilayer films with elevated layers were successfully by LBL self-assembly.

The surface morphologies of GO and RGO multilayer films were observed via AFM. As shown in Figure 6, the modified silicon substrate is covered by GO or RGO sheets after the self-assembly of the fifth layers. The root-meansquare roughness of the GO and RGO sheets is 4.613 and $4.098 \mathrm{~nm}$, respectively, indicating that the roughness of GO films decreased after reduction with hydrazine hydrate.

The microtribological behaviors of the GO and RGO multilayer films in air were evaluated by AFM. Figure 7 shows the adhesion force curves for the GO and RGO films with the different number of layers. It is obvious seen that, the adhesion force for GO or RGO films shows strong

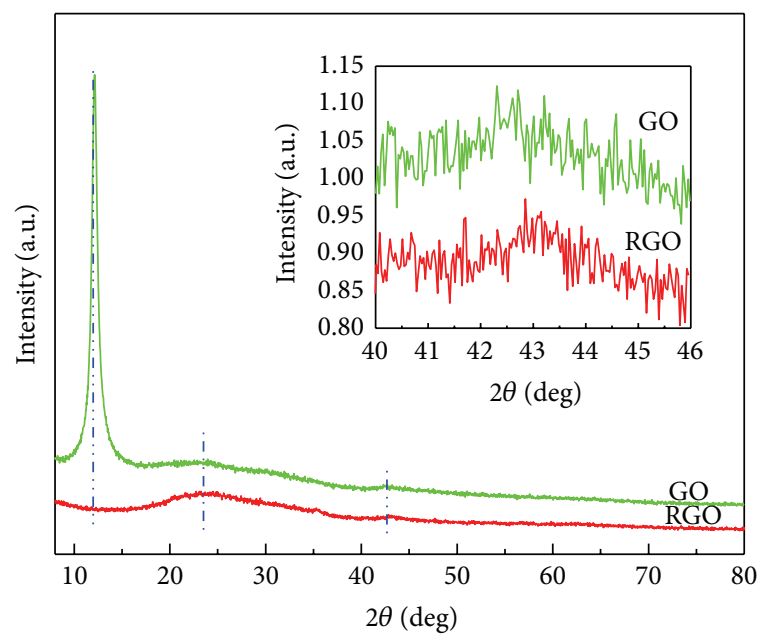

FIgURE 3: The XRD patterns of the GO and RGO sheets.

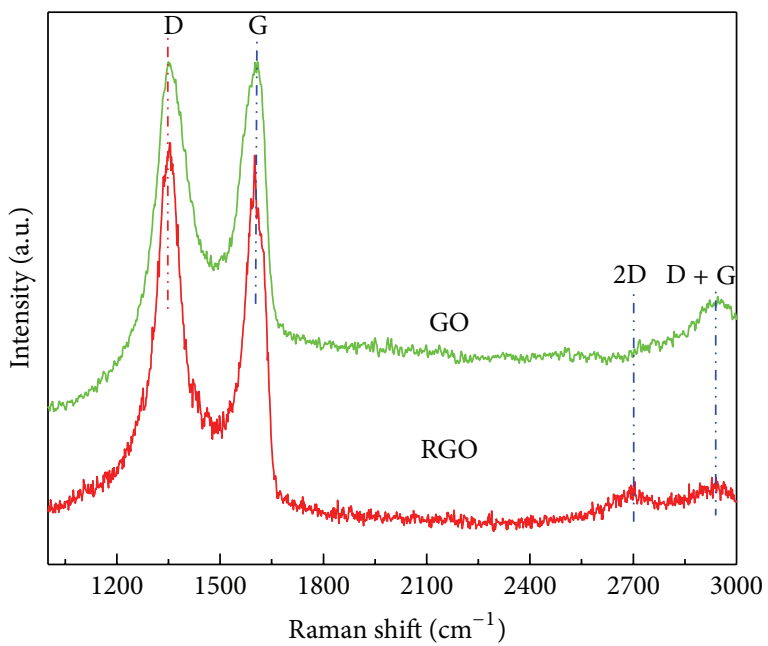

FIGURE 4: The Raman spectra of the GO and RGO multilayer films with the fifth layers.

dependence on the number of self-assembled layers, which allow a downward trend as the number of self-assembled layers increases. Moreover, in comparison with the GO films with the same self-assembled layers, the RGO films possess lower adhesion force.

In general, the adhesive force between an AFM tip and a sample surface includes the capillary force $(F c)$, as well as solid-solid interaction, Van der Waals force $\left(F_{\mathrm{vdW}}\right)$, electrostatic force $\left(F_{E}\right)$, and the chemical bonding force $\left(F_{B}\right)$ $[28,35]$. As such, $F_{\text {ad }}$ can be expressed as $F_{\text {ad }}=F_{C}+F_{\mathrm{vdW}}+F_{E}+$ $F_{B}$. Since the tip and sample sit in air for a relatively long time, no net charges are expected to remain in them. Thus, $F_{E}=0$. The chemical bonding force can also be neglected since the surfaces of the tip and the sample are saturated with chemical bonds. Thus, no ionic or covalent bonds are expected to form during contact. Therefore, $F_{\text {ad }}$ consists only of $F_{C}$ and $F_{\mathrm{vdW}}$. The capillary force $F_{C}$ is closely related to surface wettability, which can be evaluated by the water contact angles (WCAs) [36]. 


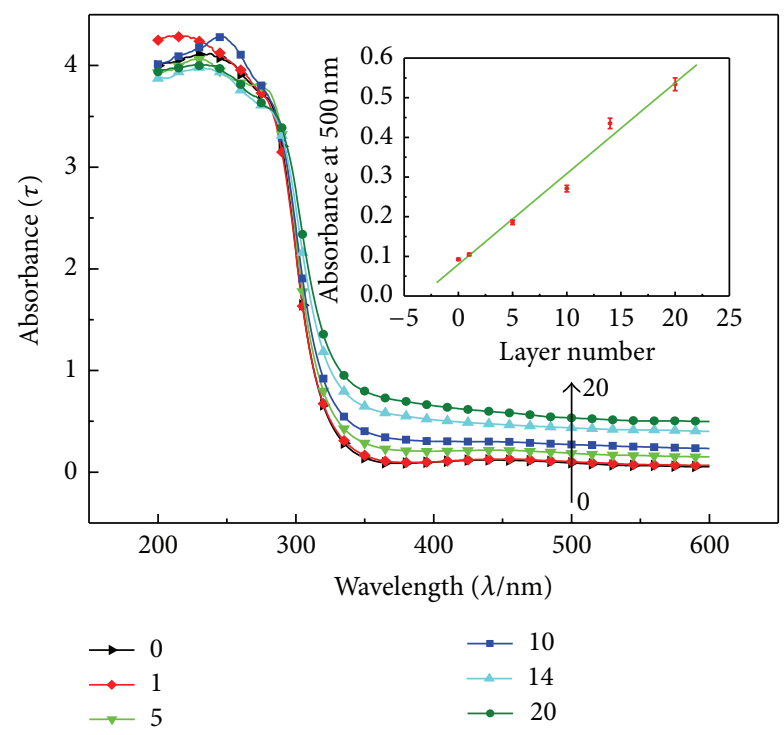

FIGURE 5: UV-vis/spectra of the RGO multilayer films on ITO glass substrates. The inset is the absorbance at $500 \mathrm{~nm}$ as a function of layer number.

The WCAs on the hydroxylated silicon surface and selfassembled films are shown in Figure 8. It shows that different numbers of layers of GO films or RGO films have almost no effect on their WCAs. However, after reduction, the WCA of GO films obviously increases from $60^{\circ}$ to $81^{\circ}$, indicating that the RGO film surface is more hydrophobic due to the decrease of abundant oxygenous groups in GO sheets after reduction by hydrazine. This point has already been proven by the XPS data of GO and RGO and explains why the adhesive force of GO films is higher than that of RGO films. Such a phenomenon may be properly understood, since adhesive force, which depends mainly on the capillary force between a tip and a surface, is dramatically lowered or even eliminated when the surface becomes hydrophobic [13].

It has been reported that $F_{\mathrm{vdW}}$ also contributes to adhesion at very low humidity [28]. The difference in $F_{\mathrm{vdW}}$ between GO and RGO is also attributed to the oxygenous groups that exist on the surfaces of the films. Compared with RGO, the surface of the GO sheets has many oxygenous groups due to graphite oxidation, resulting in strong Van der Waals forces between the edges of GO sheets and the AFM tip. Therefore, the large $F_{\mathrm{vdW}}$ may be a key factor in the relatively high adhesion of GO. From the above analyses, it could be deduced that the difference in adhesion force between GO and RGO may be attributed to the quantity of surface functional groups. In addition, the adhesion force displays a downward trend as the number of layers increases, a finding that may be due to the decrease in oxygenous groups exposed to the outer edges of the GO or RGO sheets as a consequence of the closer arrangement of the sheets.

To obtain stable friction-versus-load curves, especially on the silicon surface, a typical blunting process of a $\mathrm{Si}_{3} \mathrm{~N}_{4}$ tip via friction scanning on mica was performed in a manner similar to a previous report [37]. The friction-versus-load curves for GO and RGO films measured using blunt tips

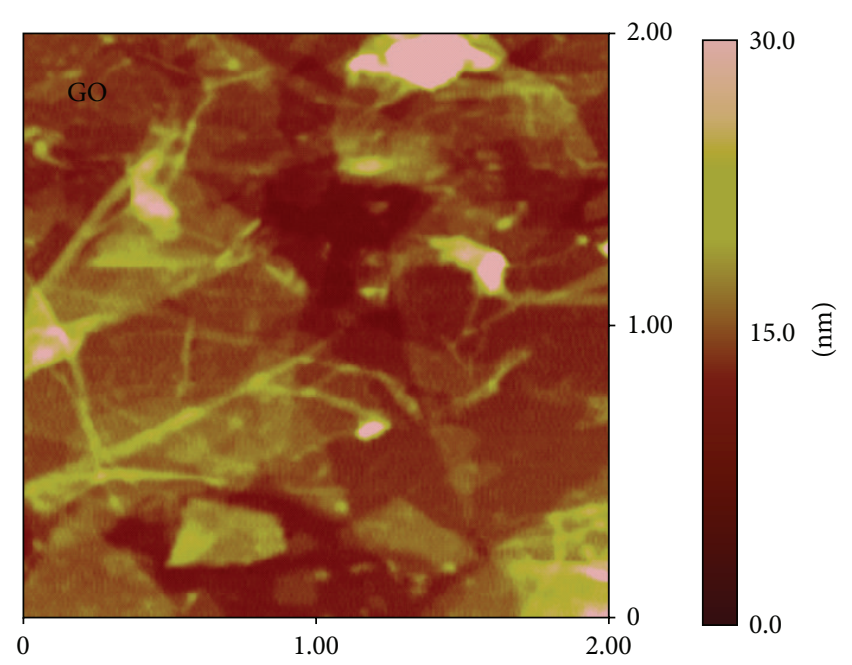

(a)

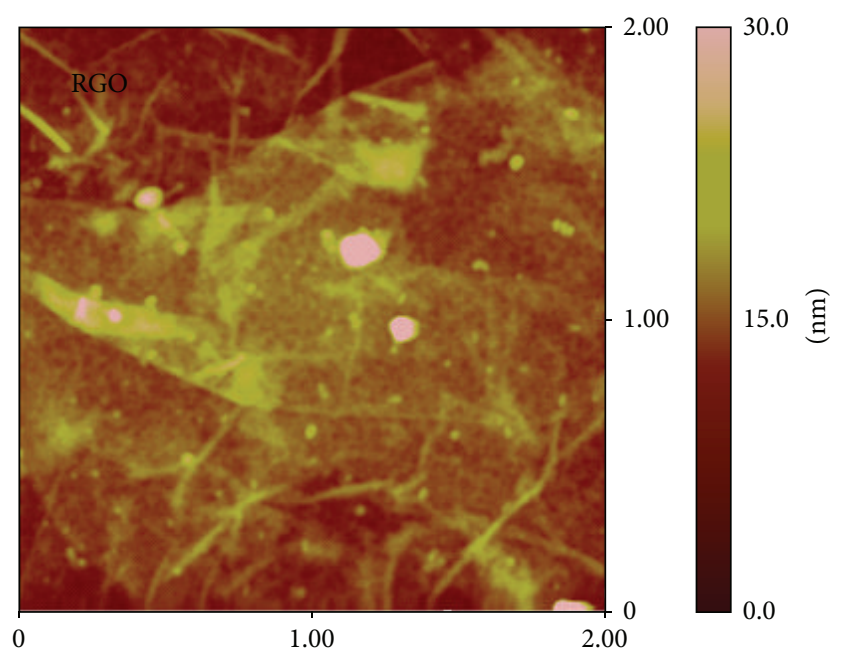

(b)

FIGURE 6: AFM images for GO (a) and RGO (b) multilayer films with the fifth layer.

are shown in Figure 9. The friction-versus-load curves show linear characteristics as the external load exceeds $360 \mathrm{nN}$, indicating that the data are in agreement with Amontons' law [26]. However, as the figure shows, not all of the friction load curves pass through the origin, suggesting that Deraguin's modification of Amontons' law should be used: $F_{L}=\mu F_{N}+F_{0}$, where $\mu$ is the coefficient of friction, $F_{N}$ is the normal force (applied load), and $F_{0}$ is the friction force when the normal force is zero $[26,38]$. According to this analysis, the gradient of a plot of the friction force against the load is equal to $\mu$. It should be mentioned that the value of $\mu$ obtained in this study is not absolutely accurate and it is not comparable with the value obtained by other authors. However, a systemic comparison among the self-assembled GO and RGO films in our investigations is highly reliable. As shown in Figure 9, the value of $\mu$ gradually decreases with the increase in number of layers of the two films. Compared with GO films with the same self-assembled layers, RGO films possess lower $\mu$ at 


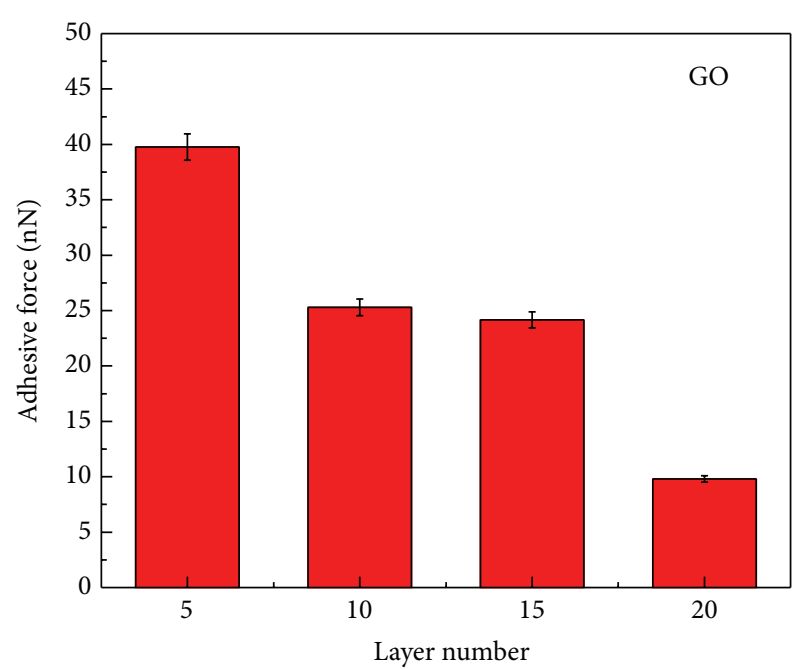

(a)

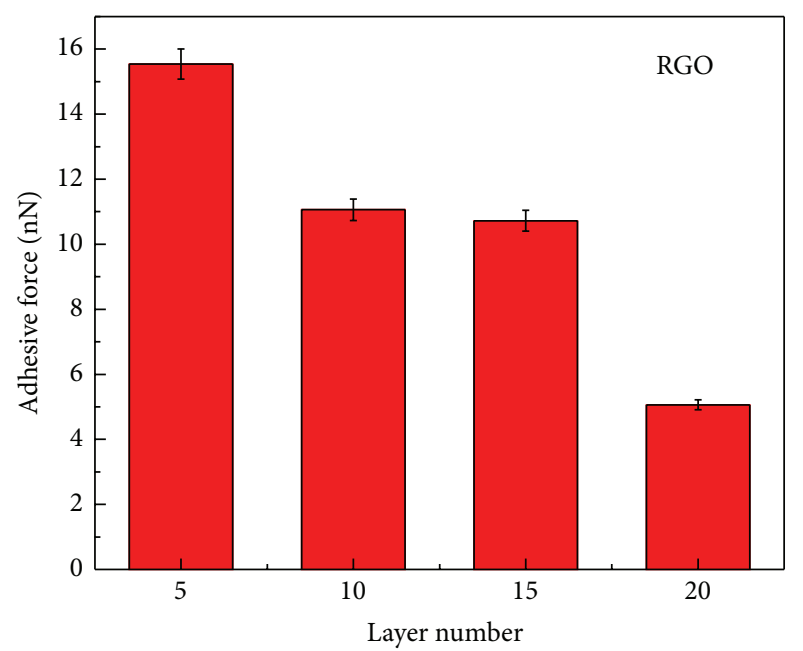

(b)

FIGURE 7: Adhesion force curves for the GO (a) and RGO (b) multilayer films with the different number of layers.

the same measurement conditions, indicating that RGO films have better lubricity. Many studies have reported that friction decreases with the reduction of adhesion on a microscopic scale [39-41]. In addition, these studies further provided a manifestation for our results. Thus, for RGO film, smaller adhesion force compared with GO film results in the lower $\mu$. It is also possible that the reduction of $\mu$ is brought about by the reduction of surface roughness after the chemical reduction, as proved by AFM images.

The possible factors that contribute to the relationship between the $\mu$ and the number of layers of GO and RGO films were analyzed. Discrepancy in the $\mu$ of GO and RGO films was also discussed. A possible explanation for the reduction in $\mu$ when the number of layers increases is that films that increasingly attach to the substrate surface via electrostatic interaction have more freedom to swing and rearrange along the sliding direction under shear stress, thus yielding smaller resistance. Interlayer sliding could have

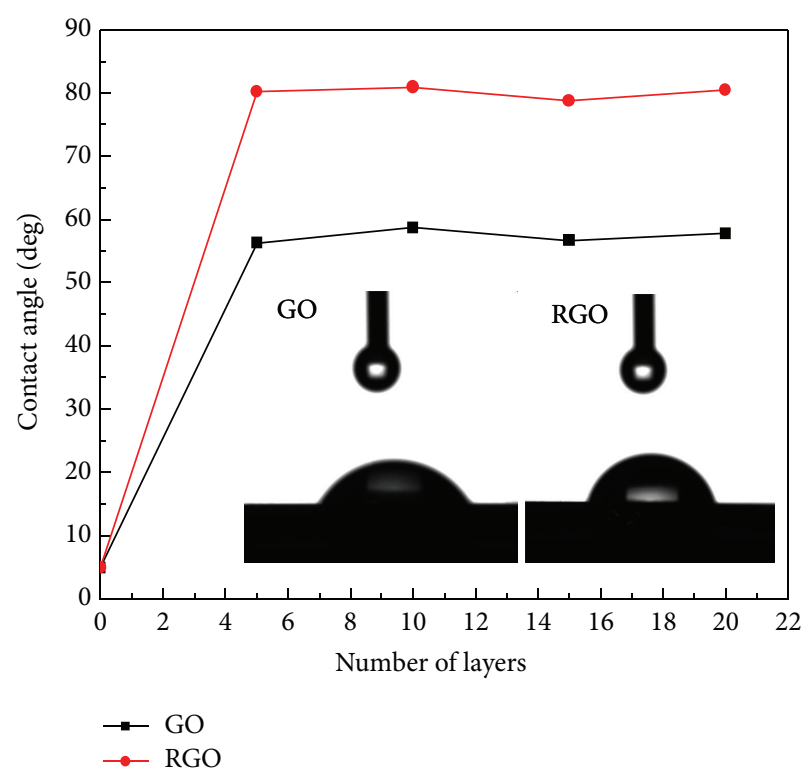

FIGURE 8: Water contact angles for the GO and RGO multilayer films with different numbers of layers. Inset is the contact angles for fifth layer.

occurred when the tip slipped across the top surface of the films in our experiments and reduced the friction of thicker samples.

Yet another possibility that may explain the mechanism of the thickness-dependent friction behavior is based on the puckering effect proposed by Lee et. al via the simple model of a tip sliding across a flexible membrane [17]. When the tip comes into contact with the top surface of the membrane, adhesion causes the sheet to easily pucker locally because of the sheet's low bending stiffness compared with its inplane stiffness [42]. The puckered geometry is modified at the front edge by tip-sheet friction, and the symmetry of the puckered region breaks and piles up mostly at the front edge of the contact; the geometry reverses when the tip changes direction. The enlarged contact area brought about by out-ofplane deformation requires more force to slide the tip forward [22]. For a thicker sheet, puckering is less prominent owing to the larger bending stiffness of the sheet [22]; therefore, friction is also lower.

\section{Conclusion}

In summary, GO and RGO multilayer films were prepared via electrostatic self-assembly on single-silicon crystal substrates. The microstructure and microtribological properties of the samples were investigated. According to the experimental results, RGO films have smaller adhesive force and lower $\mu$ compared with GO films, indicating that the former has better friction reduction properties under low applied loads in the microscopic scale. The improved tribological properties for RGO films are attributed to a decrease of abundant oxygenous groups in RGO molecules, decrease in adhesion, and a decrease of surface roughness after reduction by hydrazine. For GO films and RGO films, the adhesive 


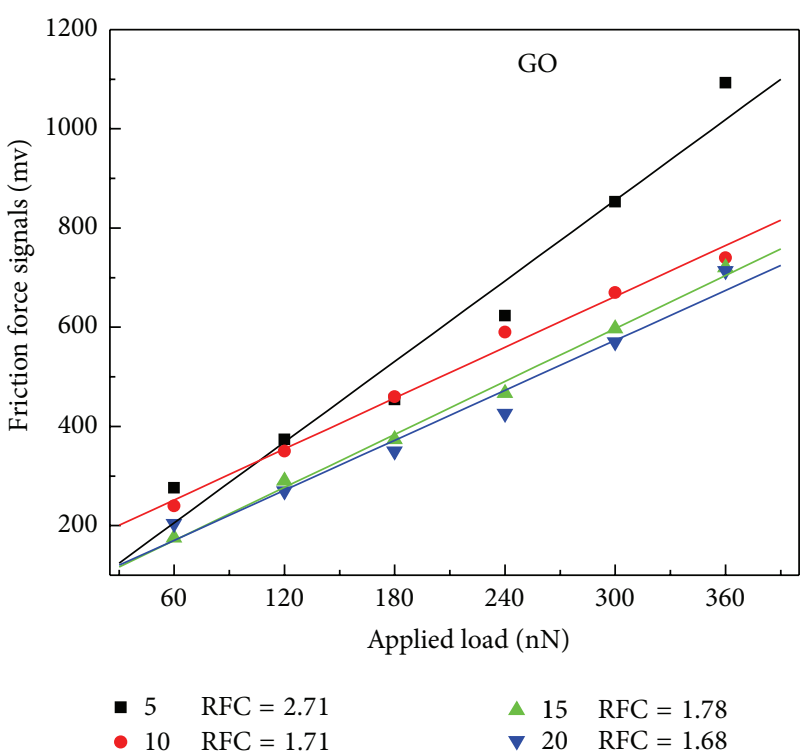

(a)

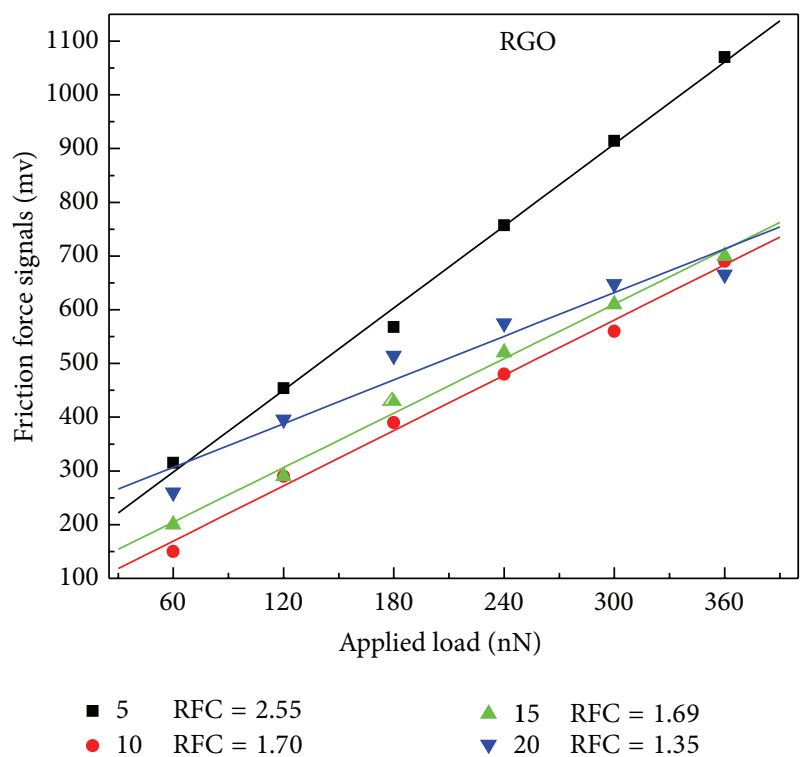

(b)

FIGURE 9: Friction-versus-load curves for the GO (a) and RGO (b) multilayer films with the different number of layers.

resistance and $\mu$ both show the downward trend as the number of layers increases. One reason is due to more freedom to swing and rearrange along the sliding direction under shear stress, thus yielding smaller resistance with the increase of number of layers. Another reason is that puckering is less prominent for a thicker sheet owing to the larger bending stiffness of the sheet; therefore, friction is also lower.

\section{Conflict of Interests}

The authors declare that they have no financial and personal relationships with other people or organizations that can inappropriately influence their works; there is no professional or other personal interest of any nature or kind in any product, service, and/or company that could be construed as influencing the position presented in, or the review of, the paper.

\section{Authors' Contribution}

The manuscript was written through contributions of all authors. Yong shouHu and Haibing Ma contributed equally to this report. All authors have given approval to the final version of the paper.

\section{Acknowledgments}

This paper was supported by the Open Fund of Key Laboratory of Mechanics on Disaster and Environment in Western China (201203) and the Natural Science Foundation of Gansu Province (1208RJYA036).

\section{References}

[1] S. M. Hsu, "Nano-lubrication: concept and design," Tribology International, vol. 37, no. 7, pp. 537-545, 2004.

[2] Y. Qin, A. Brockett, Y. Ma et al., "Micro-manufacturing: research, technology outcomes and development issues," International Journal of Advanced Manufacturing Technology, vol. 47, no. 9-12, pp. 821-837, 2010.

[3] S. M. Spearing, "Materials issues in microelectromechanical systems (MEMS)," Acta Materialia, vol. 48, no. 1, pp. 179-196, 2000.

[4] Z. Rymuza, "Control tribological and mechanical properties of MEMS surfaces. Part 1: critical review," Microsystem Technologies, vol. 5, no. 4, pp. 173-180, 1999.

[5] Y. Lvov, G. Decher, and H. Möhwald, "Assembly, structural characterization, and thermal behavior of layer-by-layer deposited ultrathin films of poly(vinyl sulfate) and poly(allylamine)," Langmuir, vol. 9, no. 2, pp. 481-486, 1993.

[6] J. C. Chen, L. Huang, L. M. Ying, G. B. Luo, X. S. Zhao, and W. X. Cao, "Self-assembly ultrathin films based on diazoresins," Langmuir, vol. 15, no. 21, pp. 7208-7218, 1999.

[7] M. Ferreira and M. F. Rubner, "Molecular-level processing of conjugated polymers. 1. Layer-by-layer manipulation of conjugated polyions," Macromolecules, vol. 28, no. 21, pp. 71077114, 1995.

[8] D. Julthongpiput, H.-S. Ahn, D.-I. Kim, and V. V. Tsukruk, "Tribological behavior of grafted polymer gel nanocoatings," Tribology Letters, vol. 13, no. 1, pp. 35-40, 2002.

[9] A. Lio, D. H. Charych, and M. Salmeron, "Comparative atomic force microscopy study of the chain length dependence of frictional properties of alkanethiols on gold and alkylsilanes on Mica," The Journal of Physical Chemistry B, vol. 101, no. 19, pp. 3800-3805, 1997.

[10] B. D. Beake and G. J. Leggett, "Variation of frictional forces in air with the compositions of heterogeneous organic surfaces," Langmuir, vol. 16, no. 2, pp. 735-739, 2000.

[11] L. Huang, G. Luo, X. Zhao, J. Chen, and W. Cao, "Selfassembled multilayer films based on diazoresins studied by atomic force microscopy/friction force microscopy," Journal of Applied Polymer Science, vol. 78, no. 3, pp. 631-638, 2000. 
[12] S. Gao and L. Yu, "Preparation and investigation of the tribological behavior of small molecular weight organic dye and polycation ultra-thin films," Acta Polymerica Sinica, vol. 1, no. 1, pp. 18-22, 2003.

[13] S. L. Ren, S. R. Yang, and Y. P. Zhao, "Micro- and macrotribological study on a self-assembled dual-layer film," Langmuir, vol. 19, no. 7, pp. 2763-2767, 2003.

[14] A. K. Geim, "Graphene: status and prospects," Science, vol. 324, no. 5934, pp. 1530-1534, 2009.

[15] K. S. Novoselov, A. K. Geim, S. V. Morozov et al., "Electric field in atomically thin carbon films," Science, vol. 306, no. 5696, pp. 666-669, 2004.

[16] I. Meric, M. Y. Han, A. F. Young, B. Ozyilmaz, P. Kim, and K. L. Shepard, "Current saturation in zero-bandgap, top-gated graphene field-effect transistors," Nature Nanotechnology, vol. 3, no. 11, pp. 654-659, 2008.

[17] H. Lee, N. Lee, Y. Seo, J. Eom, and S. Lee, "Comparison of frictional forces on graphene and graphite," Nanotechnology, vol. 20, no. 32, Article ID 325701, 2009.

[18] C. Lee, Q. Li, W. Kalb et al., "Frictional characteristics of atomically thin sheets," Science, vol. 328 , no. 5974, pp. 76-80, 2010.

[19] H. F. Yang, F. H. Li, C. S. Shan et al., "Covalent functionalization of chemically converted graphene sheets via silane and its reinforcement," Journal of Materials Chemistry, vol. 19, no. 26, pp. 4632-4638, 2009.

[20] W. Q. Zhongqing, D. E. Barlow, and P. E. Sheehan, "The assembly of single-layer graphene oxide and graphene using molecular templates," Nano Letters, vol. 8, no. 10, pp. 3141-3145, 2008.

[21] Y.-K. Kim and D.-H. Min, "Durable large-area thin films of graphene/carbon nanotube double layers as a transparent electrode," Langmuir, vol. 25, no. 19, pp. 11302-11306, 2009.

[22] J. F. Ou, J. Wang, S. Liu et al., "Tribology study of reduced graphene oxide sheets on silicon substrate synthesized via covalent assembly," Langmuir, vol. 26, no. 20, pp. 15830-15836, 2010.

[23] Y. Xu, H. Bai, G. Lu, C. Li, and G. Shi, "Flexible graphene films via the filtration of water-soluble noncovalent functionalized graphene sheets," Journal of the American Chemical Society, vol. 130, no. 18, pp. 5856-5857, 2008.

[24] M. Choucair, P. Thordarson, and J. A. Stride, "Gram-scale production of graphene based on solvothermal synthesis and sonication," Nature Nanotechnology, vol. 4, no. 1, pp. 30-33, 2009.

[25] S. Stankovich, D. A. Dikin, R. D. Piner et al., "Synthesis of graphene-based nanosheets via chemical reduction of exfoliated graphite oxide," Carbon, vol. 45, no. 7, pp. 1558-1565, 2007.

[26] N. J. Brewer, B. D. Beake, and G. J. Leggett, "Friction force microscopy of self-assembled monolayers: influence of adsorbate alkyl chain length, terminal group chemistry, and scan velocity," Langmuir, vol. 17, no. 6, pp. 1970-1974, 2001.

[27] Y.-S. Shon, S. Lee, R. Colorado Jr., S. S. Perry, and T. R. Lee, "Spiroalkanedithiol-based SAMs reveal unique insight into the wettabilities and frictional properties of organic thin films," Journal of the American Chemical Society, vol. 122, no. 31, pp. 7556-7563, 2000.

[28] X. Xiao and L. Qian, "Investigation of humidity-dependent capillary force," Langmuir, vol. 16, no. 21, pp. 8153-8158, 2000.

[29] V. V. Tsukruk and V. N. Bliznyuk, "Adhesive and friction forces between chemically modified silicon and silicon nitride surfaces," Langmuir, vol. 14, no. 2, pp. 446-455, 1998.
[30] S. Stankovich, D. A. Dikin, G. H. B. Dommett et al., "Graphenebased composite materials," Nature, vol. 442 , no. 7100 , pp. $282-$ 286, 2006.

[31] S. Stankovich, R. D. Piner, X. Chen, N. Wu, S. T. Nguyen, and R. S. Ruoff, "Stable aqueous dispersions of graphitic nanoplatelets via the reduction of exfoliated graphite oxide in the presence of poly(sodium 4-styrenesulfonate)," Journal of Materials Chemistry, vol. 16, no. 2, pp. 155-158, 2006.

[32] C. Hontoria-Lucas, A. J. López-Peinado, J. D. D. LópezGonzález, M. L. Rojas-Cervantes, and R. M. Martín-Aranda, "Study of oxygen-containing groups in a series of graphite oxides: physical and chemical characterization," Carbon, vol. 33, no. 11, pp. 1585-1592, 1995.

[33] C.-Y. Su, Y. Xu, W. Zhang et al., "Electrical and spectroscopic characterizations of ultra-large reduced graphene oxide monolayers," Chemistry of Materials, vol. 21, no. 23, pp. 5674-5680, 2009.

[34] H. Kang, A. Kulkarni, S. Stankovich, R. S. Ruoff, and S. Baik, "Restoring electrical conductivity of dielectrophoretically assembled graphite oxide sheets by thermal and chemical reduction techniques," Carbon, vol. 47, no. 6, pp. 1520-1525, 2009.

[35] M. Binggeli and C. M. Mate, "Influence of capillary condensation of water on nanotribology studied by force microscopy," Applied Physics Letters, vol. 65, no. 4, pp. 415-417, 1994.

[36] S.-L. Ren, S.-R. Yang, J.-Q. Wang, W.-M. Liu, and Y.-P. Zhao, "Preparation and tribological studies of stearic acid self-assembled monolayers on polymer-coated silicon surface," Chemistry of Materials, vol. 16, no. 3, pp. 428-434, 2004.

[37] L. M. Qian, X. D. Xiao, and S. Z. Wen, "Tip in situ chemical modification and its effects on tribological measurements," Langmuir, vol. 16, no. 2, pp. 662-670, 2000.

[38] T. T. Foster, M. R. Alexander, G. J. Leggett, and E. McAlpine, "Friction force microscopy of alkylphosphonic acid and carboxylic acids adsorbed on the native oxide of aluminum," Langmuir, vol. 22, no. 22, pp. 9254-9259, 2006.

[39] A. Noy, C. D. Frisbie, L. F. Rozsnyai, M. S. Wrighton, and C. M. Lieber, "Chemical force microscopy: exploiting chemicallymodified tips to quantify adhesion, friction, and functional group distributions in molecular assemblies," Journal of the American Chemical Society, vol. 117, no. 30, pp. 7943-7949, 1995.

[40] V. V. Tsukruk, M. P. Everson, L. M. Lander, and W. J. Brittain, "Nanotribological properties of composite molecular films: $\mathrm{C}_{60}$ anchored to a self-assembled monolayer," Langmuir, vol. 12, no. 16, pp. 3905-3911, 1996.

[41] C. D. Frisbie, L. F. Rozsnyai, A. Noy, M. S. Wrighton, and C. M. Lieber, "Functional group imaging by chemical force microscopy," Science, vol. 265, no. 5181, pp. 2071-2074, 1994.

[42] K. R. Shull, "Contact mechanics and the adhesion of soft solids," Materials Science and Engineering: R: Reports, vol. 36, no. 1, pp. $1-45,2002$. 

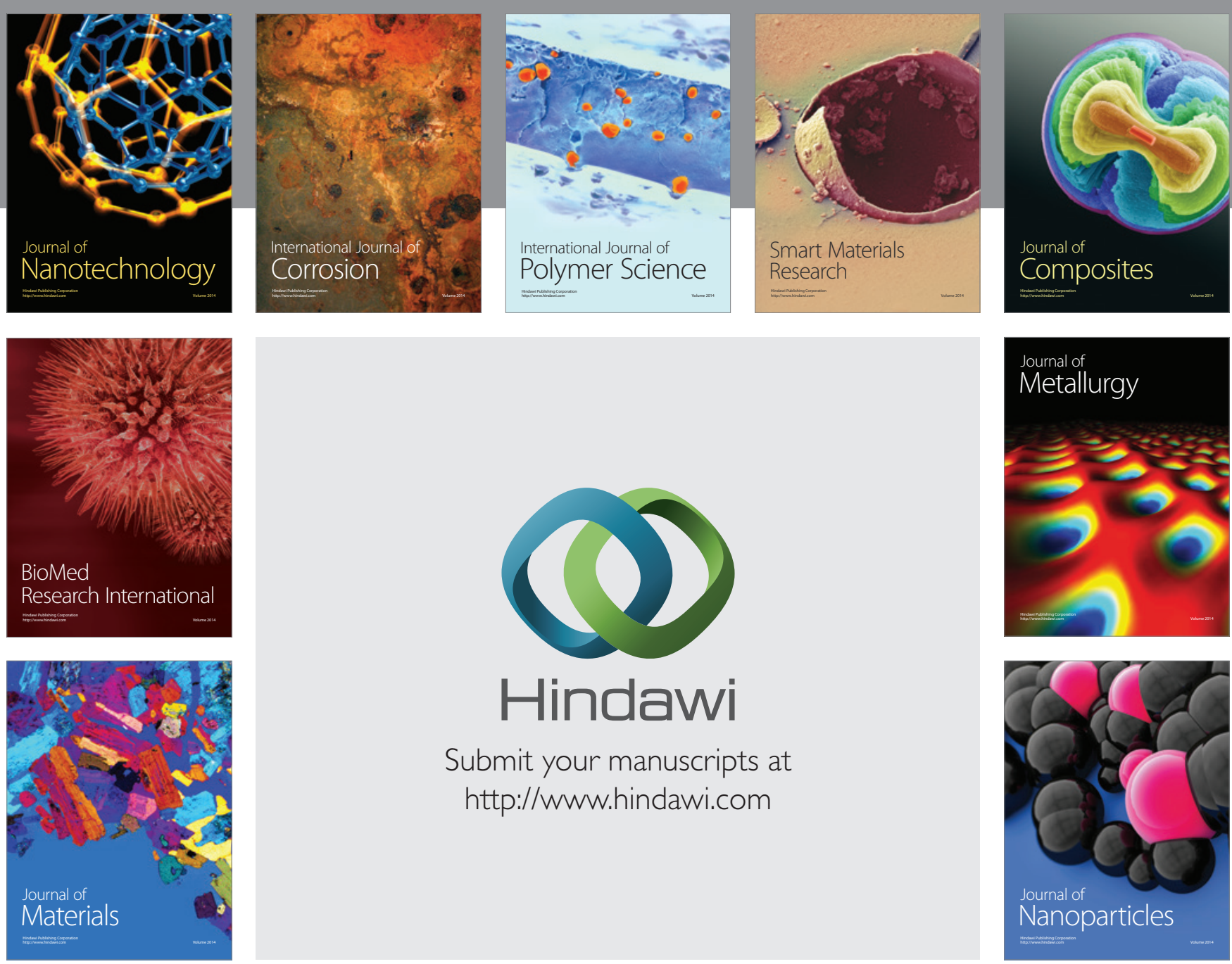

Submit your manuscripts at http://www.hindawi.com
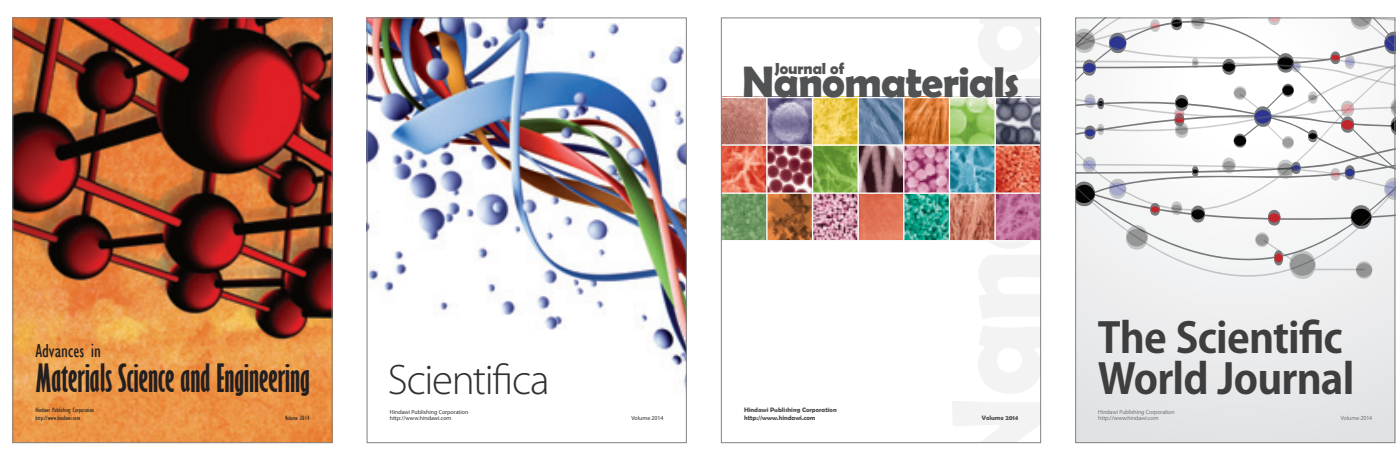

\section{The Scientific World Journal}
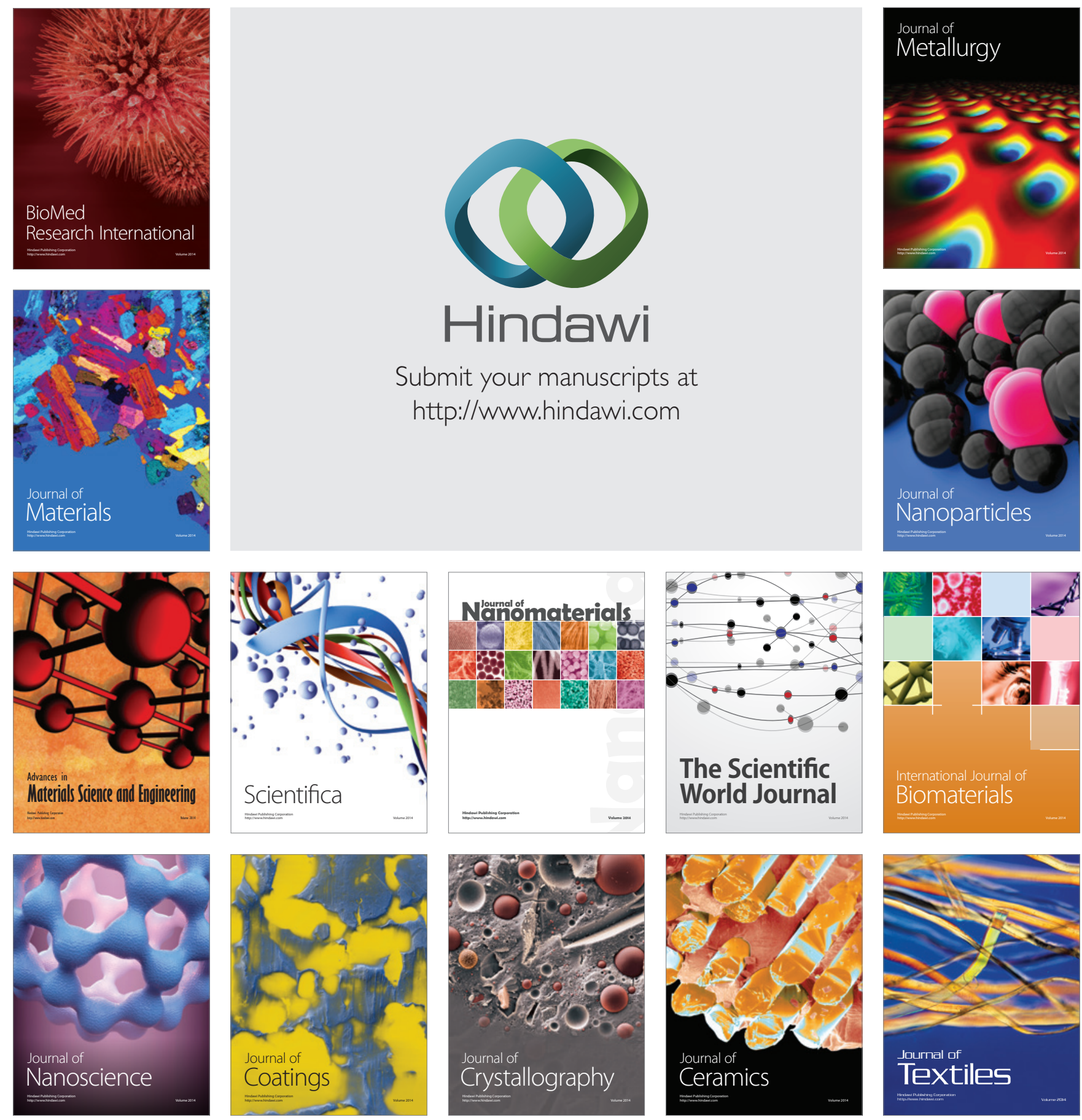